\title{
ÊTRE LÀ SANS EXISTER HEIDEGGER LECTEUR DE NATORP
}

\author{
Emanuele Mariani \\ Fundaçāo para a Ciência e a Tecnologia \\ Lisboa / Evora
}

\section{Dans quel sens celui qui existe existe-t-il?}

Qui? A l'intérieur de la Seinsfrage s'annonce une autre question: la Werfrage. La référence va principalement au $\S 64$ de Sein und Zeit, «Sorge und Selbstheit», où le «qui», c'est-à-dire le sujet, la subjectivité en tant qu'ipséité se recueille dans le Dasein, inquiet de lui-même et de son être propre'. Mais comment ce «qui» doit-il plus précisément être entendu? Strictement parlant, la question nach dem Wem échappe, au moins partiellement, au sens formel de la Seinsfrage: le «qui», dans l'incipit de Sein und Zeit, n'indique que l'un des moments du questionnement - «qui», c'est-à-dire le questionnant, celui qui pose le problème de l'être ${ }^{2}$. Dans un tout autre sens, la Werfrage du $\S 64$ reconnaît dans le «qui» le thème d'une interrogation spécifique: non pas qui demande?, mais plutôt qui est «qui»?, à savoir qui suis-je? En questionnant l'être, c'est inévitablement nous-mêmes que nous questionnons, et le $\mathrm{Wer}$, comme ces pages sembleraient suggérer, constitue un domaine autonome bien qu'en dernière instance imputable à la Frage nach dem Sein.

1 Cf. M. Heidegger, Gesamtausgabe [désormais noté GA], vol. 2, Klostermann, Frankfurt 1975 ss, p. 322. " "Ich" meint das Seiende, dem es um das Sein des Seienden, das es ist, geht ». Outre le $\S 64$, le parcours de la Werfrage au sein de Sein und Zeit se déroule à travers les étapes essentielles du $\S 9$, où la Jemeinigkeit et l'existentia définissent les principaux caractères du Dasein et, spécialement, du § 25. Cf. à ce propos GA 2, p. 153; p. 155.

2 Cf. GA 2, p. 5. 
Les motifs d'une ontologie explicitement fondée sur l'analytique existentielle du Dasein sont désormais bien connus, et il n'est point besoin ici de s'attarder sur les difficultés - déclarées du reste par Heidegger lui-même - qui caractérisent en positif aussi bien qu'en négatif l'entreprise de Sein und Zeit ${ }^{3}$. La question qui nous occupe est autre et concerne la possibilité d'affranchir la Werfrage de la Seinsfrage. Est-il possible de penser le «qui» en dehors, ou à la rigueur avant la question de l' 'être»? L'interrogation, en apparence paradoxale, a en réalité une légitimité historiographique, si l'on considère que l'«anthropologie phénoménologique», telle que le jeune Heidegger la conçoit dès le début des années vingt, précède de fait la formulation de la Seinsfrage ${ }^{4}$. La référence va cette fois la période fribourgeoise (1919-23) et, notamment, aux cours des deux semestres, d'hiver et d'été, de 1919-20 - Grundprobleme der Phänomenologie et Phänomenologie der Anschauung und des Ausdrucks - auxquels vient s'ajouter, toujours en 1919, le Kriegsnotsemester ${ }^{5}$. Le fil thématique suit l'élaboration d'une philosophie de la vie - élaboration, à vrai dire, plutôt sui generis qui prétend à la fois conférer un sens inédit à la méthode phénoménologique. Le problème du sujet, dans cette perspective, s'énonce dans les termes du selbst, le «soi», l'«ipséité mondaine», Selbstwelt, d'où

3 Il nous suffit ici de renvoyer aux questions conclusives de Sein und Zeit, ainsi qu'au caractère ouvertement inachevé de la recherche. Cfr. GA 2, p. 437; tr. fr. E. Martineau, Edition numérique hors-commerce, p 325: «Or c'est vers cela seulement [i.e. l'interprétation de l'être] que la présente recherche est en chemin. Où en est-elle arrivée? [...] Comment un comprendre ouvrant de l'être est-il en général possible à la mesure du Dasein? ». Pour une reconstruction historico-conceptuelle de l'œuvre de 1927, nous renvoyons entre autres à: T. Kisiel, The genesis of Heidegger's Being and Time, University of California Press, Berkeley 1993; J-F. Courtine, Heidegger et la phénoménologie, Vrin, Paris 1990; Id. (dir.), Heidegger, 1919-1929: de l'herméneutique de la facticité à la métaphysique du Dasein, Vrin, Paris 1996; F. Volpi, Heidegger e Brentano: l'aristotelismo e il problema dell'univocità dell'essere nella formazione filosofica del giovane Martin Heidegger, Cedam, Padova, 1976; Id., Heidegger e Aristotele, Daphne, Padova 1984; J. Greisch, Ontologie et temporalité. Esquisse systématique d'une interprétation intégrale de Sein und Zeit, PUF, Paris 1994. Pour un commentaire critique plus attentif aux apories de la Seinsfrage, voir J-L. Marion, Réduction et donation. Recherches sur Husserl, Heidegger et la phénoménologie, PUF, Paris 1989.

${ }^{4}$ On trouvera une formulation de cette hypothèse de recherche dans les travaux de S-J. Arrien. Cf. S-J. Arrien - S. Camilleri, Le jeune Heidegger: 1909-1926, Vrin, Paris 2011, p. 111-129. Cf. également S-J. Arrien, « Vie et Histoire (Heidegger 1919-1923), in Revue Philosophie, 69, 2001, pp. 51-69. Voir également C. Sommer, Heidegger, Aristote, Luther. Les sources aristotéliciennes et néo-testamentaires de Etre et Temps, PUF, Paris 2005; J. Greisch, L'arbre de la vie et l'arbre du savoir. Le chemin phénoménologique de l'herméneutique heideggérienne (1919-1923), Editions du Cerf, Paris 2000.

5 Pour une bibliographie chronologique sur l'ensemble de la production heideggérienne, nous renvoyons à C. Sommer, « Martin Heidegger: Bibliographie chronologique (1909-1976)», consultable en ligne sur le site des Archives Husserl de Paris, www.umr8547.ens.fr. 
Heidegger tire son propre champ d'investigation, en s'attaquent directement à Dilthey, Jaspers et l'Ecole de Marburg, en référence à Rickert et à Natorp. Et c'est l'Allgemeine Psychologie (1912) de ce dernier qui retiendra tout particulièrement son attention, réactivant ainsi un débat déjà extrêmement vif entre néo-kantisme et phénoménologie ${ }^{6}$.

Notre question se reformule alors comme suit: pourquoi cet intérêt pour l'Allgemeine Psychologie - intérêt renouvelé dans les trois cours fribourgeois de 1919-20? Le motif réside sans doute dans la radicalité des positions de Natorp sur le problème du «je»: celui-ci décrète en somme l'impossibilité d'un accès direct à la subjectivité. L'argument est logiquement cohérent: le je, le sujet, lorsque nous le pensons, est appréhendé comme un objet et non plus, donc, comme «je». Ainsi, l'autoréflexion, tout autant que la réflexion, n'est d'aucune aide: elle agit comme le regard de la méduse qui pétrifie tout ce sur quoi il se pose. Ne restent que les produits de la subjectivité, les objets ou, mieux, les objectivations en tant que contenus de conscience. C'est là le seul moyen qui nous est donné pour saisir le «je», ne serait-ce qu'indirectement: saisir son reflet dans les choses. Cet état de fait dépend essentiellement, aux yeux de Natorp, de la relation entre le sujet et l'objet, la Bewußtheit - ou «consciosité», pour reprendre le lexique de l'Allgemeine Psychologie - en tant que principe fondamental et irréductible. Si nous nous demandons qu'est-ce que le «je», alors le problème est déjà résolu: le «je» n'est rien d'autre que «le terme d'une relation à quelque chose dont il y a conscience» ${ }^{7}$. Dès lors, l'objectivité - le seul vrai problème de la psychologie - se définit en fonction de la relation qui s'établit à l'égard du «je». Il ne s'agit pas de voir et, par conséquent, de décrire, mais de déterminer: ce qui se donne, la «donnée», n'est rien d'autre qu'un moment partiel d'une relation plus générale, reconstructible

${ }^{6}$ Pour une reconstruction du cadre historique, qu'il nous suffise ici de savoir que pendant ces années, et plus précisément en 1918, Husserl relisait les mêmes pages de Natorp, en y trouvant des suggestions très importantes pour le développement de la méthode génétique. On connait bien, dans la Cinquième recherche logique, les critiques husserliennes à l'ego transcendantal ainsi qu'aux thèses de l'Einleitung in die Psychologie de 1888, dont l'Allgemeine Psychologie représente l'accomplissement. Tout aussi connus sont la retractatio husserlienne aux critiques initiales ainsi que les comptes rendus que Natorp écrivait aux Prolégomènes, en 1901, et aux Ideen I, en 1914 - comptes rendus auxquels il faut également ajouter, toujours en 1914, celui d'Adolf Reinach, à l'occasion de la parution de l'Allgemeine Psychologie. Il s'agit donc d'un échange intense, en grande partie public, et culminant en 1922 avec le projet d'une interprétation phénoménologique d'Aristote, le célèbre Natorp-Bericht, qui marqua l'entrée du jeune Heidegger à l'Université de Marburg.

7 P. Natorp, Allgemeine Psychologie nach kritischer Methode. Erstes Buch. Objekt und Methode der Psychologie, J.C.B. Mohr, Tübingen 1912, p. 22; trad. fr. E. Dufour - J. Servois, Psychologie générale selon la méthode critique, Vrin, Paris 2007, p. 29. 
seulement a posteriori à l'intérieur d'un processus perpétuellement en devenir. C'est en ceci que consiste la méthode de la psychologie, entendue de façon critique: reconstruire rétrospectivement, génétiquement, les traces d'un sujet qui ne se donne pas et qui se reflète tout au plus dans ce qu'il n'est pas.

Heidegger, en bon phénoménologue, s'attaquera par contrecoup à démontrer que le «je» ou, mieux, le «soi» «se donne», «est là» et d'une certaine façon «existe». Comment? A partir d'une situation donnée, individuée phénoménologiquement: la Faktizität. Il s'agit de remonter à cette «situation» pour saisirlesujetdel'expérience; pouren fairel'expérience. Et la méthode, cette fois-ci, sera celle de la Destruktion. Que donne-t-elle à voir? Et qu'obtenonsnous, une fois parvenus à l'origine, l'Ursprung de l'ipséité? La réponse passe par une critique préalable, et radicale, de l'Allgemeine Psychologie. Mais audelà de la véhémence et du style, les deux positions en apparence inconciliables présentent quelques convergences significatives: tout en critiquant Natorp, Heidegger lui accordera en fait l'essentiel ${ }^{8}$. C'est du moins la thèse que nous souhaiterions démontrer, en nous appuyant sur l'ordre de lecture qui suit: à partir des cours fribourgeois de 1919-20, nous approfondirons la position de Natorp pour ensuite revenir, dans un second temps, au jeune Heidegger dans le but d'explorer le rapport ainsi que les tensions, conceptuellement aporétiques, entre le «je», la «facticité» et l'«existence». D'un Heidegger contre Natorp nous parviendrons à un Heidegger partiellement aligné avec Natorp et, pour ainsi dire, contre Heidegger lui-même. Nous chercherons alors à saisir le sens d'un questionnement qui dans les allées et les venues de la critique et de la contre-critique nous laisse entrevoir la légitimité non seulement historiographique, mais aussi philosophique, d'une Werfrage sans Seinsfrage. Dans quel sens celui qui existe existe-t-il?

\section{Vie et histoire}

Entrons sans plus tarder in medias res: dans les deux cours de 191920, la «vie», d'après le jeune Heidegger, représente ni plus ni moins que le thème crucial de toute la philosophie - thème dont son sort dépend et qui

\footnotetext{
8 Voir à ce sujet C. von Wolzogen, " Den Gegner stark machen - Heidegger und Natorp ", in Neukantianismus. Perspektiven und Probleme, E.W. Orth - H. Holzhey (hrsg.), Königshausen \& Neumann, Würzburg 1994, p. 397-417; voir également J. Stolzenberg, Ursprung und System. Probleme der Begründung systematischer Philosophie im Werk Hermann Cohens, Paul Natorps und beim frühen Martin Heidegger, Neue Studien zur Philosophie, hrsg. von R. Bubner, K. Cramer, R. Wiehl, Göttingen 1995; " Der Weg zum Selbst. Paul Natorp und der frühe Martin Heidegger », in C. Strube (Hrsg.), Martin Heidegger und der Neukantianismus, Koenigshausen \& Neumann, Würzburg 2008, p. 51-74.
} 
nous oblige à une confrontation préliminaire avec son histoire. La raison est double et s'appuie essentiellement sur une analyse de la situation présente:

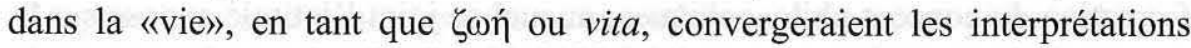
de matrice grecque, vetéro-testamentaire, néo-testamentaire, chrétienne et gréco-chrétienne qui continuent de déterminer de façon souterraine notre horizon conceptuel; constatation corroborée de surcroit par la problématique de la philosophie contemporaine, qui à son tour reconnaîtrait dans la «vie» son thème de prédilection. La philosophie de la culture, d'une part, et la psychologie, de l'autre, constitueraient les deux tendances majoritaires sur la base desquelles nous pensons encore aujourd'hui la «vie»: en vertu d'un $a$ priori qui aspire à en embrasser la totalité des manifestations et une méthode qui vise à en saisir l'élément originaire. A l'universalité de la raison fait contrepoint l'immédiateté du vécu, en instituant par là une série d'oppositions qui en délimitent le spectre conceptuel: «absolu / relatif», «transcendantal / empirique», «rationnel / irrationnel», «formel / matériel». Et c'est ainsi qu'à partir de la vie se formule le problème de l'histoire, en référence notamment aux difficultés d'un a priori historique et, en parallèle, le problème de l'ego, de son accès et de sa description. La Destruktion, nous nous limitons pour l'instant à le répéter, est la réponse à cette exigence: l'identification d'une «origine» en vertu de laquelle comprendre et dépasser le sens des oppositions. Qu'est-ce que l' «origine»? Avançons, provisoirement, une première définition: l'origine, en tant qu'arché, est ce en fonction de quoi se donne un champ de fondation et, par reflet, ce à partir de quoi se produit, généalogiquement, l'opposition elle-même. Elle est le point vers où nous procédons et d'où nous provenons. Archéologie et généalogie: l'évidente circularité ne doit toutefois pas s'entendre négativement comme une petitio principii. Il s'agit bien plutôt, comme Heidegger n'hésite pas à relever, d'une «indication formelle» qui en dénote la conceptualité spécifique: le sens de l'origine, dit de façon plus incisive, n'est que l'origine du sens ${ }^{9}$.

Trêve d'exégèse, revenons aux termes généraux du programme heideggerien. Celui-ci est déjà lisible, bien que d'une manière non proprement explicite, dans le choix des titres. Nous nous référons particulièrement au

9 Cf. M. Heidegger, Zur Bestimmung der Philosophie, GA 56/57, p. 17: «Il faut avoir présent à l'esprit, et sans ambages, le cercle qui se cache dans l'idée elle-même de science originaire». Pour un approfondissement de la question, nous renvoyons à la postface de V. Costa à la traduction italienne de M. Heidegger, Fenomenologia dell'intuizione e dell'espressione, Quodlibet, Macerata 2000, p. 173 ss. Concernant l'«indication formelle», voir également R. Rodríguez, La transformación hermenéutica de la fenomenología. Una interpretación de la obra temprana de Heidegger, Tecnos, Madrid 1997; L. Villevielle, «Heidegger, de l'indication formelle à l'existence», in Bulletin d'Analyse Phénoménologique, IX 5, 2013, p. 1-96. 
lien qui relie les Grundprobleme à une "phénoménologie de l'intuition et de l'expression». Et la même chose vaut pour le sous-titre, «Théorie de la formation du concept philosophique», auquel se joint l'intitulé apposé sur le manuscrit du cours du semestre d'été 1919-20, «Recherche sur le concept de philosophie phénoménologique». Emerge avec force, et de toute évidence, le binôme «intuition» et «expression», où se déclare l'intention d'une méthode qui continue de revendiquer la priorité de la Gegebenheit, tout en s'ouvrant sur une dimension herméneutique. Le phénomène, ce qui se donne - et c'est là l'idée fondamentale - se donne toujours et nécessairement dans un contexte déterminé de sens; au sein d'un réseau de tendances, de motivations et de significations, la Bedeutsamkeit, qui structure le monde où nous vivons; le monde qui est notre vie comme dira Heidegger - «unser Leben ist die Welt, in der wir leben» ${ }^{10}$. L'expression de cette Bedeutsamkeit n'est que la vie ellemême, où tout a pour nous une position, une valeur, un sens. Bedeutsamkeit et Gegebenheit établissent donc les coordonnées fondamentales de l'Ursprungswissenschaft, la phénoménologie, reconfigurée dans les termes d'une «herméneutique de la facticité» à la recherche d'un accès à l'ipséité mondaine - le point jaillissant d'où nous pouvons saisir et observer le phénomène de la vie.

C'est dès lors de manière similaire que s'explique le sous-titre «Théorie de la formation du concept philosophique». Le geste est ici en apparence husserlien, et s'appuie sur l'étroite convergence entre la doctrine et la méthode: la philosophie, appelée à fonder l'univers des sciences, s'autofonde, en faisant de cette auto-fondation son propre fondement. Et c'est pour cela qu'elle se distingue de toutes les autres sciences, bien qu'elle demeure à son tour, comme toute science, un savoir conceptuel. Dit plus simplement, la question de la philosophie est: pourquoi la philosophie? Sa spécificité est d'investiguer ou, pourrions-nous dire, de dévoiler ses propres motifs. Mais contrairement à ce que les Recherches logiques enseignaient jadis, par «motif» nous n'entendrons plus l'exigence d'une clarification concernant les vécus de conscience - l'Aufklärung der Erkenntnis. La différence à l'égard de Husserl est essentielle: dans la perspective heideggérienne, le «motif» indique plutôt l'origine d'où provient une expérience déterminée, un contexte de significations déterminél1. Et il devra s'entendre méthodologiquement

${ }^{10}$ M. Heidegger, Grundprobleme der Phänomenologie, GA 58, p. 33.

${ }^{11}$ Cfr. M. Heidegger, Phänomenologie der Anschauung und des Ausdrucks. Theorie der philosophische Begriffsbildung (Sommersemester 1920), GA 59, p. 185: «Destruktion ist also nicht Überleitung und Vorbereitung theoretischer Sauberkeit und Begriffsbestimmung zu Erkenntniszwecken, auch nicht als "klare" (razionalistiche) Erhellung der > übrigen Einstellungen $<-$ rationalistisch missdeutete Echtheit (vgl. Husserl !) ». 
comme un mouvement ou, mieux, un contre-mouvement à même de remonter aux sources de la significativité. Une «phénoménologie de l'intuition et de l'expression» sera donc, ipso facto, une «théorie du concept philosophique» qui recherche le motif, c'est-à-dire le champ expérientiel, à partir d'où le concept se forme.

\section{L'entrelacs onto-psycho-logique}

A ce stade, le problème qui se pose est de comprendre «comment une considération scientifique peut-elle parvenir à l'ipséité» ${ }^{12}$. Quel est le chemin qui mène à l'«origine»? La réponse, nous le savons, s'appuie sur la méthode de la Destruktion redéfinie par Heidegger dans le cours du semestre d'été de 1919-20 comme une «déconstruction orientée» ${ }^{13}$. Une manière qui ne serait selon nous pas dénuée d'intérêt pour saisir plus précisément son fonctionnement, serait de la considérer dans les termes d'une «théorie de la signification», certes sui generis, et particulièrement attentive à la dimension contextuelle ou situationnelle - «pragmatique» avant la lettre ${ }^{14}$. Or, que la Destruktion implique, outre la méthode, une théorie sinon proprement linguistique pour le moins langagière, c'est Heidegger lui-même qui le suggère. Dans le Kriegsnotsemester de 1919, nous lisons: «l'universalité des significations linguistiques [Wortbedeutung] veut dire premièrement [...] mondanité de l'expérience vécue [Welthaftigkeit des erlebten Erlebnis] $\gg^{15}$. La dimension sémantique s'avère essentiellement liée au contexte expérientiel, c'est-à-dire au contexte qui rend l'expérience possible, où par «contexte» l'on entendra l'«environnement» en tant que «monde ambiant» (Umwelt). Vécu, signification, monde ambiant. Il s'agit d'une unité complexe à caractère, pourrions-nous dire, «onto-psycho-logique», en vertu de laquelle la mondanité se manifeste dans une expérience sémantiquement articulée et la signification se définit comme l'expression d'un vécu ambiant ayant son origine dans le monde. A titre illustratif, mettons-nous à la place du jeun Privatdozent en nous rapportant au célèbre exemple de la «chaire». Comment se décrit l'expérience de celui qui, en entrant dans l'amphithéâtre, voit la chaire d'où il tiendra son cours? Voici une situation possible: «Je vois la chaire aussitôt en une fois; et je ne la vois pas de façon isolée, mais je vois la chaire comme trop haute pour

\footnotetext{
${ }^{12}$ Cf. GA 58, p. 94.

${ }^{13}$ Cf. GA 59, p. 34.

${ }^{14}$ Cf. V. Costa, Postfazione, in M. Heidegger, Fenomenologia dell'intuizione e dell'espressione, cit., p. 172. Cf. également GA 59, p. 35.

${ }^{15}$ GA 56/57, p. 117.
} 
moi. J'y vois un livre dessus comme quelque chose qui me dérange $[\ldots]\rangle^{16}$. L'expérience serait naturellement tout autre si c'était «un paysan de la Forêt Noire» qui entrait dans l'amphithéâtre ou «un noir du Sénégal». Placés dans un environnement inconnu, dans un monde étranger, l'un comme l'autre ne verraient pas la chaire, mais sans doute plutôt un banc en bois derrière lequel se réfugier, un abri, ou peut-être ne percevraient-ils même qu'une superficie plate ou un ensemble de couleurs. Il n'y aurait de commun aux deux situations que le fait que dans les deux cas quelque chose est vu. Dans les deux cas, il y a quelque chose, quelque chose se donne - es gibt-, même sans savoir exactement «quoi». Heidegger peut alors commenter: «dans l'expérience vécue de voir la chaire, quelque chose se donne à moi [gibt sich mir etwas] à partir d'un monde ambiant immédiat [aus einer unmittelbaren Umwelt]», pour ensuite conclure:

Ce qui est significatif est ce qui est primaire [das Bedeutsame ist das Primär], il se donne à moi [gibt es mir] immédiatement sans un détour intellectuel qui passerait par une conception des choses. Vivant dans un monde ambiant, toujours et partout cela a pour moi une signification [bedeutet es mir], c'est donc mondain [es ist alles welthaft], "cela mondanise» [«es weltet $》] .^{17}$

Es gibt, es bedeutet, es weltet - «cela se donne», «cela signifie», «cela mondanise». La séquence tripartite, exception faite du néologisme «es weltet», ne manque pas de mettre à profit et radicaliser une indication jadis husserlienne, en donnant lieu à une analyse qui se veut rigoureusement phénoménologique. L'intention, la signification d'un mot - par exemple la «chaire» - comme l'enseignait Husserl, trouve confirmation dans un remplissement intuitif, en présence de la chose elle-même. En la pointant du doigt, nous pouvons dès lors dire: «la chaire». Et c'est ainsi que nous la percevons: comme une «chaire», à savoir comme un objet d'usage qui remplit une fonction donnée, et auquel l'on attribue de surcroît une signification sociale. Nous ne voyons pas une superficie plate, une extension colorée, un ensemble d'éléments successivement reconfigurés dans un objet déterminé. Au contraire, nous en saisissons aussitôt la signification, en la contextualisant dans un environnement tout aussi significatif: l'amphithéâtre, l'université, l'école, etc. L'expérience ne se borne pas à donner forme à un amas de sensations d'après les catégories de l'intellect. L'acquis phénoménologique est plutôt autre, et concerne la donation, la Gegebenheit, d'un monde ambiant: la perception, pour le dire avec Husserl, est déjà apperception et recèle en soi un surplus, qui nous permet

\footnotetext{
${ }^{16}$ GA $56 / 57$, p. 70 ss.
}

${ }^{17}$ GA 56/57, p. 73. 
de percevoir ce que nous percevons exactement comme nous le percevons. L'intuition, en d'autres termes, présente un caractère significatif qui ne fait plus correspondre à la Bedeutungsintention un simple état de choses (Sachverhalt); le corrélat objectif se redéfinit dans les termes d'un Bedeutungsverhalt ou, comme le remarque Heidegger, un Lebensverhalt - un "état significatif», un «substrat vital». La conséquence la plus remarquable est alors la suivante: la significativité assume la teneur d'un caractère mondain, en définissant la modalité originaire à travers laquelle le monde nous apparaît-Bedeutsamkeit, Weltcharakter. Ici réside la valeur, si l'on veut, "pragmatique» de l'analyse: dans un rapport essentiel au monde, sur lequel vient se greffer la question de l'origine en tant qu'origine du sens - l'origine qui n'est rien d'autre que sens, ce d'où le sens provient. En un mot, Herkunft ${ }^{18}$. Mais, nous demandons-nous, quel monde serait un monde dans lequel ce seraient les significations, et non pas les objets, ce que nous rencontrerions en premier? Quel monde serait un monde où tout serait significatif? Un monde essentiellement stratifié où l'existence, la nôtre, se concrétise temporellement, historiquement, à travers une accumulation constante, en fonction de certaines attitudes et motivations. Un monde dans lequel la stratification porte inévitablement en soi le danger d'une perte: la poussière du temps occulte la signification des choses, le sens de la vie. Des mots nous ne retenons que le concept vide, sans forcément réactiver le contexte qui en a produit le sens. La question de l'origine émerge donc d'une exigence de compréhension face à une situation de crise, qui engage le présent - le présent où la question se pose. Ainsi se détermine le point de départ à partir duquel la méthode débute: das Verblassen der Bedeutsamkeit, «l'effacement de la significativité». Et la pratique de la destruction s'oriente par conséquent vers une désambiguïsation préliminaire.

Considérons à ce propos le concept d'«histoire», par lequel nous pouvons concevoir la science qui étudie le passé; un domaine de faits établis; la tradition. Nous disons, en outre, que l'histoire est magistra vitae, qu'un homme a une histoire riche en aventures ou qu'une histoire triste lui est arrivée ${ }^{19}$. Où le principe directeur à même de régler et d'expliquer cette équivocité réside-t-il? Heidegger introduit ici un autre concept sous le titre de Situationphänomene, qui décrirait la matrice à partir de laquelle s'engendrent les différentes configurations de sens ${ }^{20}$. Comprendre une «signification» c'est comprendre le contexte dans lequel elle a été produite et tout contexte implique, constitutivement, certaines relations, certaines tendances et motifs imputables

\footnotetext{
${ }^{18} \mathrm{Cf}$. à ce propos GA 56/57, p. 117.

${ }^{19}$ Cf. GA 59 , p. 43 ss.

${ }^{20}$ Cf. GA 59, p. 35.
} 
en dernière instance à une expérience fondamentale qui circonscrit le caractère d'une «situation» donnée. La destruction, en tant que «déconstruction orientée» s'avance donc vers une double direction: archéologique, en vue des motivations sous-jacentes au contexte; généalogique, en fonction d'une progression qui va de l'originaire au non-originaire, de l'authentique à l'inauthentique.

Toujours à titre illustratif, considérons maintenant un concept plus banal, tiré des pages de Sein und Zeit. Nous pouvons dire: «le marteau est trop lourd», et entendre par là qu' « il n'est pas léger», que «son maniement exige force et rend fatiguant son usage». Nous pouvons par ailleurs dire qu'il a un poids et des propriétés déterminées; qu'il exerce une certaine pression et que sans appui il tombe ${ }^{21}$. Du marteau comme ustensile nous passons à un autre type de considération, à une autre attitude qui l'envisage comme une chose corporelle soumise aux lois de la physique. Il s'agit de situations évidemment hétérogènes, et hétérogène est également la compréhension du milieu ambiant: un lieu, tel qu'une table, une boîte à ustensiles ou des coordonnées spatiotemporelles. Nous sommes toujours face au même marteau, et pourtant pas de la même manière. Comment se décrit le passage d'une attitude à l'autre? Quelles sont les limites? L'expérience, en premier lieu, directe, se modifie graduellement jusqu'à s'énoncer comme un jugement apophantique - «le marteau est...». Un étant donné est ainsi thématisé et «la thématisation rend objective»: l'étant devient une «simple présence», une chose, là où la chose en tant que Dinglichkeit annonce le motif dominant de la science, l'attitude théorétique, qui trouve historiquement sa réalisation la plus exemplaire dans la mathématisation de la nature. Voici le résultat auquel les cours fribourgeois de 1919-20 parviennent déjà, en avance sur les analyses de 1927: tout jugement, toute attitude, au-delà des différences spécifiques, recèle en soi une expérience effective, même sous une forme cachée ou entièrement raréfiée, même si le «rythme de la vie» paraît s'arrêter, même si le «tapis de la vie» se fait inéluctablement invisible sous nos pieds ${ }^{22}$. La fameuse devise «zu den Sachen selbst !» trouve ainsi une confirmation inédite, en se déclinant dans une tout autre perspective: sans intuition - comme aurait dit Husserl - la signification se vide en devenant purement symbolique et, privés de contexte - ajouterait sans doute le jeune Heidegger -, les mots perdent leur sens, leur pourquoi. L'effacement de la Bedeutsamkeit n'est rien d'autre que cette «perte», où le

${ }^{21}$ Cfr. GA 2, § 69 b.

${ }^{22}$ GA 58, p. 69. La métaphore est employée par Heidegger lui-même, s'inspirant ici de Stefan Georg. 
contenu de l'expérience se détache du contexte originaire, se dévitalise, en se rendant disponible à d'autres fins et d'autres fonctions.

\section{Du moi qui vient à l'idée}

Les questions adressées à l'Allgemeine Psychologie reprennent en substance ce type de considérations. De quelle manière la vie, en tant que qu'expérience vivante, se rend-elle accessible au regard de celui qui l'observe - le regard de la philosophie? Quel est le chemin qui conduit au psychique, à la subjectivité? Pour Natorp, on le sait, décrire le moi signifierait l'objectiver, et l'objectiver reviendrait à le transformer en quelque chose qu'il n'est pas. Mais sans «moi» peut-on encore parler de psychologie? Et quel serait l'objet de son enquête? Faudrait-il renoncer à la possibilité d'une science du vécu? C'est précisément de cela que traite l'Allgemeine Psychologie: faire de la psychologie une science et, plus précisément, une science de l'«origine»; la psycho-logie en tant que logos qui recherche les principes premiers, l'arché, l'a priori de la psyché, qui se livre à la recherche de ce qui est fondamental et, par là, se définit comme «générale». Fondement, principe, a priori, origine: c'est par ces termes que se définit la tâche de cette science - sui generis bien sûr -, dans la tentative de rétablir l'unité originaire de la conscience, malgré l'impossibilité d'un accès direct. Ce qui est subjectif ne peut pas s'exprimer en des termes objectifs. Aucune analogie avec le monde extérieur n'est utile: nous ne ferions rien d'autre qu'appliquer subrepticement au vécu des catégories qui ne sont pas les siennes. Pensons à l'exemple de la rétine, qui ne peut pas se voir tant qu'elle n'est pas reflétée dans un miroir $^{23}$. La conscience, de la même manière, ne peut être perçue qu'au travers de ses contenus. Et c'est seulement en devenant l'objet d'une auto-perception, qu'elle devient «conscience de soi»-conscience dans laquelle il faudra toutefois distinguer un «je pur», originaire, qui perçoit, et un «je empirique», dérivé, ou plutôt perçu, où la pureté du «je» ne pourra jamais devenir objet de perception, sous peine de régression à l'infini. Comment résoudre cette aporie? Le geste de Natorp consiste pour l'essentiel à identifier dans ce présupposé le principe de la solution, en faisant de la limite une donnée positive: dans toute forme d'objectivité se dissimule, en filigrane, un rapport à la subjectivité; l'objet devient l'indice d'une subjectivité latente, dans laquelle il se constitue en tant qu'objet. Non ne dirons plus alors «objet», mais à proprement parler «projet» - ce qui se place face à un sujet inaccessible en tant que tel, et sans lequel

${ }^{23}$ P. Natorp, Allgemeine Psychologie, cit., p. 30; trad. fr. p. 53. 
il n'aurait aucun sens ${ }^{24}$. Rien n'existe indépendamment de cette corrélation originaire en fonction de laquelle les deux pôles - objectif et subjectif - ne sont que deux moments abstraits. L' «être conscient de quelque chose» doit par conséquent s'entendre sous la forme de l'infini: le temps verbal qui exprime le mieux le sens de cette relation, où la relation est le caractère fondamental de la conscience qui ne peut en aucun cas être hypostasiée en substance, et par là, substantivée sur le plan grammatical. Dès lors, si l'on se demande en quoi consiste, plus précisément, cette relation, la réponse devient tautologique: la relation « est quelque chose d'ultime qui, précisément, comme tel, ne peut ni ne doit être davantage expliqué ou réduit» ${ }^{25}$. La possibilité d'une première identification du je émerge ainsi, quoiqu'en négatif: celui-ci, tout à fait hétérogène à ses propres contenus, est nécessairement présupposé dans toute forme de relation - comme le revers de la médaille. Dans tout ce que nous percevons - et nous ne percevons rien d'autre que des objets - une relation est implicitement présente, une relation qui plus est asymétrique, où le je se place comme l'autre terme d'un rapport à quelque chose dont il a conscience; comme ce qui a conscience de l'autre, sans que quoi que ce soit d'autre puisse avoir conscience du je. Qu'est-ce que le «je»? La réponse, de nouveau, est logiquement cohérente: un présupposé, une supposition, ou, mieux, une idée nécessaire qui nous permet de saisir la concaténation des vécus, et qui garantit un fondement et donc une unité à notre expérience.

Cet argument - cela vaut la peine de le relever - répond à la célèbre critique husserlienne formulée dans la Cinquième recherche logique contre l'hypothèse d'un je transcendantal, hypothèse que Natorp défend, en l'énonçant très exactement comme suit: la relation, en tant que caractère essentiel de la conscience, est toujours une relation à..., et appartient en premier lieu aux contenus psychiques; elle est une connexion entre contenus différents. $\mathrm{Si}$, toutefois, la conscience était identique, et de façon indifférenciée, aux diverses formes de connexion à chaque fois en acte, il s'avérerait problématique de comprendre comment il est possible d'être conscient de la diversité des contenus qui se composent en unité. Considérons l'écart qui réside entre l'unité d'une perception - je vois, par exemple, un merle qui vole - et une perception en tant qu'unitaire, où j'ai conscience de son déroulement - et qui me permet de percevoir le vol d'un merle, en en distinguant les différents moments. La connexion entre les contenus perçus implique, sur un autre niveau, l'unité de certaines fonctions, de certaines synthèses déterminables a priori, telles que par exemple la synthèse temporelle à même de reconfigurer la linéarité d'une

${ }^{24}$ Ivi, p. 67; trad. fr. p. 92 .

${ }^{25}$ Ivi, p. 27; trad. fr. p. 50. 
succession en un «avant» et un «après». Le je, en ce sens, n'est rien d'autre que le principe de cette unification, et il doit rigoureusement se distinguer de l'unité dont nous sommes à chaque fois conscients - l'unité phénoménale, c'est-à-dire le «je empirique», donné effectivement dans tout acte de conscience. Voir un merle en vol signifie «je vois un merle qui vole». Tout autre fonction implique, en revanche, le présupposé qui nous permet de comprendre la composition des différents moments dans une synthèse supérieure, en faisant émerger l'unité dans la diversité et, par reflet, la diversité dans l'unité. Ceci est le «je pur», la possibilité de l'unification, le fondement ultime, c'est-à-dire l'idée en tant qu'unité idéale, qui rassemble en soi la totalité des phénomènes sans pouvoir pour autant se manifester. Comme, du reste, Natorp le reconnaît lui-même:

Nous aboutissons à une distinction semblable à celle de l'idée platonicienne et de ses participants. L'idée n'apparaît pas en tant que telle, mais il ne faut pas moins la supposeret cette supposition est nécessaire - puisque, sans la supposition de l'idée, le phénomène lui-même ne serait pas fondé et ne pourrait par conséquent pas avoir de validité. ${ }^{26}$

La référence à Platon doit évidemment se lire en un sens kantien: l'unité de l'objet se fonde sur l'unité de l'expérience, laquelle est à son tour fondée sur une synthèse originaire, définie dans les termes d'un a priori - l'«aperception transcendantale», pour le dire avec Kant. Le je est donc cette synthèse qui poursuit Natorp - «se présente dans la consistance de la loi par laquelle toutes les connexions, à l'infini, sont déterminées ou déterminables en général, par conséquent sans être données, c'est-à-dire a priori» ${ }^{27}$. Idée, unité, loi: le «je», en tant que principe, œuvre comme le fondement qui transcende la complexion à chaque fois délimitée des contenus, en imposant à la méthode de la psychologie un sens éminemment dynamique. Le je est l'illimité, il est l'idée sans limites qui traverse la progression infinie de ses productions, il est la forme qui structure le devenir continu d'où toute objectivation se produit. Se profile ainsi l'entrelacs d'un chiasme où tout ce qui est - en un mot, l'être - vient à se concrétiser dans une dé-limitation de l'illimité. L'être en tant qu' " être devenu» et le devenir en tant que «genèse», étymologiquement entendue dans le sens d'une «production» d'où jaillit le mouvement continu de la vie. C'est ici que réside le caractère authentiquement «idéaliste» de cette psychologie transcendantale, en opposition avec une conception bien plus superficielle de l'idéalisme. Que l'objet soit nécessairement un objet pour la conscience représente, au fond, une thèse triviale, déjà impliquée par la complémentarité des termes mis en rapport - «subjectif» et «objectif». La

${ }^{26} I v i$, p. 36 ; trad. fr. p. 59.

${ }^{27}$ Ivi, p. 38 ; trad. fr. p. 61. 
question concerne plutôt le mode dans lequel se constitue, relativement à la conscience, l'objectivité; ce que signifie cette objectivitée ${ }^{8}$. Le maître-mot de l'idéalisme tel que l'entend Natorp - et Husserl, nonobstant les divergences, serait d'accord sur ce point - est constitution ${ }^{29}$. Il s'agit alors de remonter aux sources de la subjectivité pour saisir le sens de toute objectivation, où le sens correspond au mode, au comment de sa constitution dans la conscience. Le comment, et non le qu'est-ce que, est la question de l'idéalisme à la recherche d'un accès à la sphère des vécus. Voilà pourquoi la «genèse» signale Natorp - recèle en soi «l'expression la plus profonde de l'origine» ${ }^{30}$, où l'origine doit s'entendre dans une acception rigoureusement idéale: l'origine se déploie sous la forme d'une idée qui en tant que telle n'existe pas; elle n'est pas un fait, mais rend compréhensible et donc fonde la totalité des phénomènes. «L'idée est loi, explication, non pas substance»; elle est «la pleine et concrète expression de l'apparaître» et signifie le «concret ultime», le «vécu originaire», le «devenir continu», l'«illimité» qui ne peut jamais s'exhiber que dans les termes d'une limite idéale, inatteignable et nécessaire, dont l'achèvement est toujours à venir ${ }^{31}$.

Voici la conséquence de taille sur laquelle l'Allgemeine Psychologie

${ }^{28}$ Ivi, p. 203-204; trad. fr. p. 232-233. Natorp, de façon significative, poursuit ainsi: «Il en résulte une figure déjà plus profonde, pour ainsi dire plus noble, de l'idéalisme. Ce n'est pas simplement la "conscience" (selon laquelle nous avons en général conscience de quelque chose), mais c'est l'unité de la conscience qui constitue dans l'unité de la loi l'unité de l'objet - voilà comment on pourrait énoncer la formule fondamentale de cet idéalisme».

${ }^{29}$ Pour un approfondissement du rapport Husserl-Natorp nous renvoyons à l'introduction de M. Ferrari, «Natorp tra Kant e Husserl. Il neokantismo di Marburgo di fronte alla fenomenologia ", ainsi qu'à la postface de G. Gigliotti, « Ricostruzione o riduzione. L'eredità kantiana della sintesi nel neokantismo e nella fenomenologia », in P. Natorp, Tra Kant e Husserl. Scritti 1887-1914, Le Lettere, Firenze 201, p. 5-68; p. 161-190. Voir également le monumental I. Kern, Husserl und Kant, M. Nijhoff, Den Haag 1964; G. Gigliotti, Avventure e disavventure del trascendentale. Saggio su Cohen e Natorp, Guida, Napoli 1989; S. Besoli - M. Ferrari - L. Guidetti (a cura di), Neokantismo e fenomenologia. Logica, psicologia, cultura e teoria della conoscenza, Quodlibet, Macerata 2002; S. Luft, Reconstruction and Reduction: Natorp and Husserl on Method and the Question of Subjectivity, in R.A. Makkreel - S. Luft (ed.), NeoKantism in Contemporary Philosophy, Indiana University Press, Bloomington and Indianapolis 2010, p. 59-91; C. Morujão, « A recepção das Investigações Lógicas por Paul Natorp ", Phainomenon. Revista de Fenomenologia, n. 16/17, 2008; É. Dufour, Paul Natorp. De la "Psychologie générale" à la "Systématique philosophique", Vrin, Paris 2010.

${ }^{30}$ P. Natorp, Allgemeine Psychologie, cit., p. 76; trad. fr. p. 100.

${ }^{31}$ Cf. ivi, p. 39. Toujours de Natorp, voir également le célèbre Platos Ideenlehre: eine Einfürhung in den Idealismus, Leipzig, Dürr 1903; voir également Über Platos Ideenlehre, Reuter und Reichard, Berlin 1914, et en particulier l'introduction de V. Politis à la traduction anglaise, P. Natorp, Plato's Theory of Ideas, Academia Verlag, 2004, p. 11. 
se consolide. Qu'est-ce que la subjectivité? Une perpétuelle production de sens qui ne saurait s'exposer pour ce qu'elle est originairement à moins d'invertir le mouvement normal des choses dans un contre-mouvement qui, en progressant à rebours, reproduit génétiquement et indirectement un autre mouvement, où l'être se fluidifie en devenir, où tout s'écoule et où l'origine s'appelle vie. " Même en faisant toute la route» - décrétait jadis Héraclite - «on ne peut trouver les limites de l'âme, tant elle a un logos profond $»^{32}$. Natorp s'approprie sciemment l'antique devise, en redéfinissant la méthode qui conduit aux profondeurs du «fondement sans fond», où - et le point est décisif - la donnée originaire n'est pas la psyche, mais son logos. Ce qui se donne, se donne comme un «problème». La seule concession au principe phénoménologique de la Gegebenheit se borne à cela: donné n'est que le problème d'une psyche inaccessible, se soustrayant à tout regard. Il reste le logos que nous ne pouvons déterminer ou reconstruire qu'en vertu de ce que la psyche n'est pas. À la lumière du logos, la psyche s'épanouit et c'est dans le logos qu'elle se donne à connaître, dans l'idée qui embrasse tout phénomène sans pourtant se confondre avec le phénomène lui-même. L'objet et la méthode de la psycho-logie sont dès lors un tout: bien qu'à proprement parler elle n'existe pas, la subjectivité devient «vraie» en fonction de l'idée qui la rend nécessaire. Comment? À travers un processus de reconstruction qui dévoile $a$ posteriori la légalité d'un a priori. «Reconstruire», en d'autres termes, signifie comprendre, et comprendre signifie porter à la connaissance, puisque c'est dans la pensée que la vie se conserve, en se concrétisant en "conscience». «C'est seulement dans la pensée» - conclut sans ambages Natorp - «que le moi devient conscient comme tel $\rangle^{33}$.

\section{Le je transfiguré}

La critique «destructrice», on l'aura compris, s'attaque à l'inflexion idéaliste de la méthode, visant à démasquer le contexte et les motifs qui s'en trouvent à la base. Comment s'explique cette superposition en vertu de laquelle l'accès à la subjectivité, la méthode, coïncide essentiellement avec la subjectivité elle-même? La réponse, pour le jeune Heidegger, réside dans l'institution de l'enquête, sous-tendue par une «pré-conception» opératoire, et non suffisamment explicitée: l'idée de constitution, entendue comme «idée guide» au problème du je. «Ce n'est qu'à l'intérieur d'une problématique

${ }^{32}$ Héraclite, fr. 45 Diels-Kranz, cit. par Natorp dans Allgemeine Psychologie, cit., p. 290; trad. fr. p. 323.

${ }^{33}$ P. Natorp, Allgemeine Psychologie, cit., p. 245; trad. fr. p. 277. 
dominée par l'idée de constitution» - observe, en fait, Heidegger - «que le je est le fondement du problème $)^{34}$. Dans l'Allgemeine Psychologie, nous le savons, la constitution indique l'œuvre de la conscience, où «rien se donne comme isolé», "où même la séparation est une forme de relation», et où tout est en dernière instance imputable à un ensemble de connexions, régies par la légalité d'un a priori - en un mot, la «synthèse originaire» que Kant définissait en son temps par le syntagme «je pense». Quelle est alors l'expérience concrète, si effectivement il y en a une, qui motive cette institution ? Quelle forme de vie se cache derrière une telle vision du «je»? Voici l'objection: «La non-pensabilité du "je" met à jour de la manière la plus évidente que son sens réside dans son être référé au penser»; «le "je" est déterminé exactement à partir du penser - et précisément en pensant jusqu'au bout le penser $»^{35}$. Ici se trouve le supposé talon d'Achille de la psychologie nach kritischer Methode: derrière le «je pense» se cache un «je pensant», un «je théorétique» réduit à l'expression d'une loi, une logique à laquelle tout est en principe assignable.

Avec la pré-conception de la constitution tout - ce qui est objectif et ce qui est subjectif-est prédestiné à être un ensemble de relations, une objectualité ou une choséité dans le sens le plus ample du terme. Tout tombe victime de ce sort. ${ }^{36}$

Penser, c'est-à-dire déterminer, universaliser, fixer, assurer (Sicherung) ${ }^{37}$. Dans la disposition de la méthode vient ainsi se réfléchir un autre primat ou, plutôt, une prédominance, d'autant plus significative que latente, imputable à une radicalisation de l'attitude théorétique. Il s'ensuit une tractation de la subjectivité «théorétiquement défigurée», «dé-vitalisée» qui, en privilégiant le sens formel de l'a priori, occulte les traces d'un refoulement: «en s'opposant à ce qui est anthropologique, on a finit par rejeter hors de la philosophie tous les problèmes concernant l'existence ${ }^{38}$. La responsabilité incombe entièrement à cette forme de pensée, incapable de se reconnaître dans son être «vie» et, donc, incapable de saisir le concret, l'originaire - l'individuum. Le remède, il est à peine besoin de le dire, passe par il lebendinge Rückgang in den Ursprung. Littéralement, «la régression vitale vers l'origine», qui redéfinit la «tâche ultime du travail phénoménologique»: le retour aux contextes de

${ }^{34}$ GA 59, p. 131.

${ }^{35}$ GA 59, p. 132: «[Das Ich] bestimmt sich gerade vom Denken her, und zwar vom Zuendedenken des Denkens ».

${ }^{36}$ GA 59, p. 142-143: « Mit dem Vorgriff der Konstitution ist alles - Objektives und Subjektives - zum Beziehungszusammenhang, zu einer Sachlichkeit oder Dinglichkeit im weitesten Sinne prädestiniert. Alles verfällt diesem Schicksal ». Cf. également GA 56/57, p. 87.

${ }^{37}$ GA 59, p. 139.

${ }^{38}$ GA 59, p. 25; p. 66. 
sens authentiques, d'où l'on peut tirer les structures qui rendent intelligible le domaine de la vie. L'analyse de ces contextes se fonde, plus précisément, sur une série de "caractères descriptifs» obtenus en vertu d'une donnée fondamentale: la relation de perméabilité réciproque entre Ich et Welt. S'ensuivent les figures du Selbstwelt, de l'Umwelt, du Mitwelt qui structurent le tissu de connexions, le sol à partir duquel toute expérience prend forme. Et elles se rassemblent, en dernière instance, sous le concept plus général de «quotidienneté» - l'Alltäglichkeit, en tant que temporalité originaire de la Faktizität $^{39}$. Le passage qui mène de l'immédiateté d'une expérience à l'acquisition d'un habitus pour finalement aboutir à sa raréfaction, le jugement théorétique, s'explique de cette manière: à la lumière des connexions entre les différents contextes qui n'indiquent rien d'autre que l'effacement progressif de la Bedeutsamkeit - Erfahrungszusammenhang, Verfügbarkeit, Habitus, Wissenschaft. Traduisant le transcendantal en praxis, le geste heideggerien rend dès lors possible la remontée à la «racine anthropologique», où nous découvrons un nouvel aspect de l'a priori, anthropologiquement conçu, qui distingue cette version inédite de la phénoménologie de toute autre forme d'anthropologie ${ }^{40}$.

Mais cela suffit-il à assurer un accès à l'ipséité? Suffit-il de montrer que derrière toute théorie se cache une pratique pour élire l' «anthropologique » domaine privilégié de l'enquête? C'est le même Natorp qui formulera ces objections, en devançant les critiques soulevées par le jeune Heidegger. Encore une fois, le raisonnement est cohérent et s'appuie sur le rapport entre les deux types d'attitude - théorétique et non-théorétique. Comment s'explique le passage de l'un à l'autre, sans admettre un travail d'objectivation, sousterrain et silencieux, déjà à l'œuvre dans le domaine de l'originaire? Au demeurant, qu'il y ait continuité semble même être présupposé par le sens génétique de la Destruktion ${ }^{41}$. Afin qu'une expérience puisse avoir lieu, il

39 Voir également les analyses ultérieures que Heidegger livre dans son dernier cours de la période fribourgeoise: Ontologie (Hermeneutik der Faktizität), GA 63.

${ }^{40}$ Pour un approfondissement de la question «anthropologique», en relation avec le statut de la phénoménologie chez Heidegger aussi bien que chez Husserl, voir en premier lieu la conférence de E. Husserl, "Phänomenologie und Anthropologie », in Husserliana [désormais noté Hua], Kluwer Academic Publishers, vol. XXVIII, Aufsätze und Vorträge (1922-1937), ainsi que les articles pour l'Encyclopedia Britannica in Hua IX, Phänomenologische Psychologie, p. 237-254; p. 256-257. Concernant Heidegger, voir GA 2, § 10. Pour un commentaire critique nous renvoyons à D. Souche-Dagues, « La lecture husserlienne de Sein und Zeit », in E. Husserl, Notes sur Heidegger, Les Éditions de Minuit, Paris 1993.

${ }^{41}$ Le problème sera repris et approfondi dans le $\S 69 \mathrm{~b}$ de Sein und Zeit, où Heidegger placera à la base du passage entre les deux types d'attitudes, non-théorétique et théorétique, le schéma «si... alors», redéfini successivement en termes de «réflexion». 
est bien nécessaire que «quelque chose», préliminairement, soit donné et par conséquent soit représenté, objectivé - autrement, comment pourrait-on déceler dans l'objectivation un processus de croissante dé-vitalisation? Les «représentations communes» - l'expression se trouve dans l'Allgemeine Psychologie - ou les représentations de la vie de tous les jours, comme dirait Heidegger, se distinguent par leur caractère fluide, indéterminé, non encore accompli, elles peuvent s'avérer plus ou moins «obscures» par rapport à la clarté et la rigueur des représentations scientifiques. Il s'agira toutefois de représentations, c'est-à-dire de formes d'objectivation qui s'appliquent indistinctement à toute sorte d'activités psychiques. «Pour la psychologie» conclut donc Natorp - «la représentation non scientifique, dans la mesure où elle veut et pense pourtant toucher les choses effectives, est sur la même ligne que la représentation scientifique» ${ }^{42}$. La même objection - limitons-nous à le mentionner - se laisserait formuler en termes husserliens. Nous nous référons particulièrement à la Cinquième recherche logique, où Husserl décompose le concept brentanien de «représentation» en deux moments distincts, la matière et la qualité, pour ensuite les regrouper en une seule classe - les actes objectivants. La fonction de ces actes intéresse transversalement tout le domaine de la conscience et consiste, pour l'essentiel, à fournir à chaque espèce de vécu la représentation de l'objectivité à laquelle il se réfère. Husserl, pour sa part, établit que le «vécu intentionnel est un acte objectivant ou se fonde sur un tel acte» ${ }^{43}$. D'ailleurs, que l'intentionnalité soit toujours intentionnalité de... peut se comprendre également d'une telle manière: tout ce qui est, doit pouvoir se décrire dans les termes d'une objectualité et l'objectualité se définit comme le corrélat d'un acte en soi objectivant ou qui inclut en son sein un acte objectivant.

Quelle est donc la réponse d'Heidegger? Le but est de montrer que le type d'intentionnalité à l'œuvre dans la dimension non-théorétique est bien autre, et tout autre est également la teneur expressive des jugements qui en découlent. Dire de la «chaire» qu'elle est de «couleur marron», qu'elle est une «caisse en bois» ou une «chose» implique une attitude radicalement incompatible avec le jugement qui en exprime l'éventuel inconfort ou, à la rigueur, la gêne que sa vue occasionne. A cet égard, nous connaissons la distinction entre le «caractère apophantique» et le «caractère herméneutique» du logos - distinction qui trouve déjà dans ces cours une première formulation. De l'«als» expérientiel qui structure le monde de la Bedeutsamkeit il faudra distinguer l' «als» de la «caractérisation conceptuelle ou générale», typique de l'attitude théorétique.

${ }^{42}$ P. Natorp, Allgemeine Psychologie, cit., p. 196; trad. fr. p. 226.

${ }^{43}$ E. Husserl, Hua XIX/1, p. 514. 
Et le même vaut pour l'etwas, le corrélat objectif de toute espèce d'intentionnalité, déterminable en fonction des divers degrés de l'expérience vécue. Le «quelque chose en général», entendu formellement comme le «x» auquel la conscience se réfère, ne doit pas se confondre avec le «quelque chose vital et pré-mondain», dans lequel s'exprime «un moment essentiel de la vie» ${ }^{44}$. Que la conscience soit, par définition, toujours et nécessairement «conscience de...» ne nous oblige pas à voir dans l'objet «quelque chose de théorisé et radicalement dévitalisé». Au contraire, si l'on peut faire l’expérience de tout, alors tout est assignable au sens d'un vécu nous permettant de comprendre les choses à partir de la vie elle-même. Formellement, tout est vie. Et c'est ici que réside l'erreur de Natorp:

En absolutisant d'une telle façon ce qui est logique, Natorp ne peut voir les représentations des choses qu'à la guise d'un stade préliminaire et rudimentaire d'une véritable position logique de l'objet. ${ }^{45}$

Le «panlogisme» auquel s'assujettit la méthode de l'Allgemeine Psychologie contraint le non-théorétique à se concevoir comme prethéorétique, c'est-à-dire en fonction de la dimension théorétique, en rabattant la sphère originaire du vécu sur une «objectivation élémentaire» ${ }^{46}$. «Ce dont l'on peut faire l'expérience en général» se vide dans le «quelque chose en général»: de l'Erlebbares überhaupt on passe à l'etwas überhaupt que Heidegger définit comme Weltlose, «sans monde» ou Weltfremde, «étranger au monde». Le même ordre de considérations s'applique naturellement au «je», le corrélat subjectif de l'expérience. Revenons à l'exemple de la chaire: trouve-je quelque chose comme un «je», se demande Heidegger, dans ce genre de vécu?

En voyant la chaire je suis là avec mon je, intégralement [bin ich mit meinem vollen ich dabei], mon je y résonne [es schwingt mit] [...], c'est mon propre vécu [ein Erlebnis eigens für mich], et c'est ainsi que je la vois; pourtant il ne s'agit pas d'un processus [Vorgang], mais d'un événement [Ereignis $]^{47}$.

Dans l'acte de perception, je suis pleinement absorbé par ce que je vis. Le je s'actualise perceptivement, sans se confondre pour autant avec les choses

${ }^{44}$ GA 56/57, p. 112.

${ }^{45}$ GA 56/57, p. 108.

${ }^{46}$ GA 56/57: « Eine originäre Erlebnissphäre, wie die des Umwelterlebnisses, würde er [sc. Natorp], wenn überhaupt anerkennen, so nur wiederum als rohe Objektivierung ».

${ }^{47}$ GA 56/57, p. 73. Cf. ibid.: « Nur in dem Mitanklingen des jeweiligen eigenen Ich erlebt es ein Umweltliches, weltet es, und wo und wenn es für mich weltet, bin ich irgendwie ganz dabei ». 
qui lui font face. Il est un écart qui se décrit par métaphore dans les termes d'une «résonance» - à l'image d'une onde acoustique. Le rythme de la vie résonne et dans cette résonance œuvre une rétention qui confère au je un caractère vivant, temporel. Vibrant au contact du monde, le je vis et là où la résonance s'estompe, il se dévitalise graduellement jusqu'à ce que le rythme de la vie ne s'arrête et l'expérience se rigidifie dans une attitude objectivante qui pense, constate, thématise. Relevons alors une conséquence de taille, sur laquelle aboutit la méthode de cette "science de l'origine»: l' " empirique» et le «transcendental» se traduisent dans le sens d'une autre opposition, entre le «vivant» et le «théorétique», une opposition dont la logique œuvre par contrariété, et non par contradiction et consent un passage graduel entre les extrêmes. Il en va de même pour la distinction entre «psychique» et «physique», entre «subjectif» et «objectif», entre «dedans» et «dehors», qui se reformulent en termes d'«événement» et de «processus». Le «vécu» - observe Heidegger est ce qui m'est propre (eigen); c'est mon vécu; hic et nunc, chaque fois unique et temporellement irrépétible; il arrive (ereignet); je m'approprie (ereigne es mir) et il est donc «événement» (Ereignis), contrairement à l'itérabilité d'un processus qui se produit là dehors, parmi les choses du monde ${ }^{48}$. Erlebnis, eigen, ereignen, Ereignis: en opposition explicite avec le régime de la choséité, le caractère d'《événement» engage l'intégralité de la dimension subjective et se place de ce fait en-deçà de la distinction entre l' «authentique» et l'«inauthentique» - distinction, celle-ci, non encore thématisée ni pleinement opératoire dans les cours de 1919-20. Et c'est bien ici que réside l'élément le plus problématique, pour ne pas dire aporétique, qui empêche de cantonner trop aisément l'argument de Natorp, comme nous allons le voir.

\section{L'expérience du soi}

Reformulons notre question: l'événementialité, ainsi comprise, suffit-elle à garantir au je son principe d'individuation? La question du qui trouve-t-elle une possible réponse? Contrairement aux résultats de la réflexion ultérieure, il est à noter - et non sans surprise - qu'à ce stade de l'analyse, Heidegger perçoit dans le qui l'affectation d'une posture théorétiquement contaminée, une posture qui, en prétendant rendre accessible l'ipséité, la réifie en «égoïté», c'est-à-dire dans l'équivalent subjectif de la choséité ${ }^{4}$. Rien qu'en demandant

${ }^{48}$ GA $56 / 57$, p. $75-75$.

49 GA 56/57, p. 89-90: « Das historische Ich ist ent-geschichtlicht bis auf einen Rest von spezifischer Ich-heit als Korrelat der Dingheit, und es hat nur im Nachgehen des Theoretischen sein Wer, d.h. nur "erschlißbar") ?!"». 
«qui?», nous serions du coup exposés à une volonté de savoir objectivant le «je», l'immobilisant. Quaestio mihi factus sum - quelle est alors la juste question, si effectivement il en est une? De quel accès disposons-nous pour comprendre qui est le je, qui suis-je, alors que le simple fait de le demander suffit déjà à nous barrer la route? Ici émerge l'aporie la plus redoutable, qui finit par redonner crédit, par contrecoup, aux objections de Natorp: tant que tout, dans la sphère subjective, est indistinctement «événement», l'ego n'est pas en soi distinguable de ses contenus. Percevoir un paysage, formuler un jugement, aimer, haïr, avoir du goût, des préférences, réaliser un projet, avoir une famille, des amis, voyager, travailler, etc. A tout moment, le je coïncide, voire est ses mêmes contenus et il n'est pas de vécu sans contenu - il n'est pas de je sans monde. C'est donc ailleurs, à savoir dans le monde que paradoxalement nous regardons pour décrire le je, dont les caractères s'avèrent variables en fonction des circonstances et des différents moments de la vie.

Voici, alors, le problème: en renonçant à la pureté du transcendantal, le rapport de perméabilité entre Ich et Welt, pour le dire en termes heideggériens, menace d'éclipser le sens concret, individualisant, de l'ipséité, dont il ne resterait rien d'autre qu'une résonance, que les traces d'une accumulation qui se stratifie dans une quotidienneté, une habitude, une pratique de vie. Datum essentiellement négatif, le je occuperait le foyer obscure d'une relation qui se décrirait dans les termes d'une unité synthétique ou, si nous préférons, d'une résonance rétentionnelle et vibrante au contact des choses. Pour reprendre la formulation critique de Natorp, le je ne serait que le terme d'un rapport à quelque chose dont il y a conscience et coïnciderait, jusqu'à se confondre, avec ses contenus, à savoir les objectivations en tant que produits d'une subjectivité en soi inaccessible et impossible à expliquer d'avantage. Anthropologiquement nous sommes ce que nous faisons et dans ce que nous faisons - nous glosons à notre tour -, s'exprime une tendance vers la généralisation, d'autant plus problématique que c'est l'individuation qui est ici en cause. Une difficulté supplémentaire s'ajoute ainsi: dans la dimension quotidienne inhérente à toute forme de vie est en vigueur le sens d'une généralité et, donc, d'une normalité latente qui concerne de fait la sphère de l'expérience originaire, la sphère du soi qu'on rencontre dans le monde - l'ipséité mondaine qui vit «normalement», suivant le cours silencieux de ses habitudes. Si c'est dans la quotidienneté qu'il faut rechercher l'origine, l'originaire s'avère dès lors généralisable, voire universalisable. Et ce qui se perd paradoxalement c'est le moment du «concret»-1'individuum, le se ipse. Voilà la conséquence à laquelle nous nous exposons: le sens «authentique» de l'ipséité ne réside pas dans la quotidienneté. Ce n'est pas dans la répétition d'une habitude que je me 
trouve moi-même. Au contraire, c'est seulement en me trouvant moi-même que je peux découvrir le sens d'une habitude; ce n'est qu'en me trouvant que je peux m'habituer à moi-même. Conscient de l'aporie, Heidegger reformule l'objection comme suit:

Ce qui est habituel c'est ce qui est significatif dans la modalité de l'ipséité mondaine, mais ce n'est pas ce qui constitue son existence. ${ }^{50}$

Un écart entre facticité et existence semblerait alors poindre, donnant lieu à une autre situation dans laquelle l'ipséité n'est plus assignable au sens d'une genèse, une situation dans laquelle elle n'est plus définissable à la lumière de ses caractère mondains, voire de ses contenus. Littéralement, une situation où le je est, ou mieux, se donne sans monde. Bien qu'en apparence paradoxale, c'est là la voie qui paraît s'esquisser dans les séminaires de 1919-20, où est introduit, quoique succinctement, un concept plutôt singulier et dont le traitement concis en rend la lecture particulièrement difficile: la «significativité pure de l'ipséité mondaine ${ }^{51}$. Son occurrence se trouve dans une analyse du Vollzug, l'«effectuation» ou «accomplissement effectif», concept tout aussi important qui contribue à l'exhibition méthodologique du «phénomène», entendu dans sa «totalité de sens». Face à tout type d'expérience-Heidegger argumente - nous pouvons poser essentiellement trois questions: qu'est-ce qui est vécu; comment est vécu ce qui est vécu; comment s'effectue le sens de l'expérience elle-même ${ }^{52}$. Les deux premières questions répètent visiblement la distinction canonique entre le was et le wie - la «chose», c'est-à-dire le contenu et le «comment» de la référence intentionnelle. Dans la troisième, en revanche, il est introduit un élément de nouveauté: en redoublant la question du «comment», le Vollzug spécifie le sens de l'intention sur la base du contexte situationnel ${ }^{53}$. L'attitude théorétique, négativement comprise, sert d'exemple: réduisant le monde ambiant à une choséité, l'indétermination naturelle de l'étant se cristallise dans une forme dévitalisée et disponible à d'autres usages - établir, généraliser, universaliser, calculer, etc. Dans un contexte donné, émergerait ainsi un mode dominant de l'ipséité, dont il serait possible de détecter le degré de vitalité. Dans le comment de l'intention qui

${ }^{50}$ GA 59, p. 84: «Das Habituelle ist selbstweltlich bedeutsam, aber nicht existenzausmachend».

${ }^{51}$ GA 59, ibid.: « reine selbstweltliche Bedeutsamkeit»,

${ }^{52}$ Pour une thématisation plus ample du Vollzug, nous renvoyons à GA 60, Phänomenologie des religiösen Lebens, p. 63; trad. fr. J. Greisch, Phénoménologie de la vie religieuse, Gallimard, Paris 2011. La référence va particulièrement au $\S 13$, significativement intitulé «L'indication formelle».

${ }^{53} \mathrm{Cf}$. à ce propos 1' « Avvertenza » de F. Volpi, in M. Heidegger, Fenomenologia della vita religiosa, Adelphi, Milano 2003, p. 15-30. 
s'effectue serait constatable un sens directif, une attitude à même d'englober les deux premiers moments, le contenu et la référence, au sein d'une plus ample configuration de sens. Ce que nous obtenons - signale Heidegger c'est la façon dont la vie humaine $a$ elle-même; la façon dont j'ai moi-même. Dans l'effectuation de mes intentions, je suis moi-même qui s'exprime; c'est le sens de mes intentions qui détermine qui je suis. Non pas, donc, ce que je fais, mais comment je suis; la question du qui, pour le dire autrement, consiste à demander si et dans quelle mesure j'ai moi-même. Et c'est dans cet avoir, dans ce «s'approprier», avant même d'être, que réside l'existence. Au qui répond un comment.

Le problème, à ce stade, est de comprendre plus précisément comment s'effectue l'identité du selbst. Certainement pas en vertu d'une réflexion qui transformerait le sujet encore une fois en objet - la convergence avec Natorp est pleine. Mais comment, alors? En faisant directement l'expérience du «soi». L'accès au soi passe par une expérience, et toute la difficulté est de ne pas en réduire le sens à un objet de réflexion ${ }^{54}$. Naturellement, cela n'empêche pas qu'un acte réflexif puisse, et à la rigueur doive avoir lieu. Il ne faut toutefois pas confondre les deux sens du génitif: le «soi» peut s'entendre, d'une part, objectivement - pensons, par exemple, à l'examen de conscience, au fait d'être conscient de soi - et, de l'autre, subjectivement, comme celui qui fait l'expérience d' «être soi-même» ou, pour le dire avec l'expressivité du langage courant, qui «se réalise». Ce qui importe c'est de relever que le principe de donation est ici autre: le soi se donne par effectuation, en vivant concrètement et de façon directe une certaine situation, un certain contexte de significations. La fonction du Vollzug s'accompagne, par conséquent, d'un autre moment: le «renouveau» (Erneuerung). En s'effectuant dans ses possibilités propres, l'ipséité se renouvelle; elle vit authentiquement selon une modalité originaire de la vie. «Je m'approprie moi-même»; «je suis affecté par moi-même et j'existe dans une effectuation renouvelée [ich selbst von mir selbst und immer neue betroffen bin im erneuten Vollzug bin] ${ }^{55}$. Le Vollzug n'indique pas une expérience réitérable, d'où l'on peut tirer un habitus. "L'être affecté par soi-même croît d'une certaine manière», et conduit au «rejet de toute trace définitive» ${ }^{56}$. L'effectuation a donc le caractère de l'unicité, de la nonrépétabilité et chaque fois c'est «comme la première fois [wie zum ersten Mal], chaque fois c'est toujours plus désorientant $»^{57}$. Telle est la teneur par laquelle

\footnotetext{
${ }^{54} \mathrm{Cf}$. à ce propos GA $2, \S 25$, p. 115.

${ }^{55}$ Cf. GA 59, p. 84.

${ }^{56}$ Ibid.

${ }^{57}$ Ibid.
} 
se décrit l'expérience du soi: à chaque fois que le soi existe, c'est «comme la première fois», et en existant - voilà le point qui nous intéresse le plus - il suspend toute relation avec le monde. «Ce moment du "comme la première fois"», confirme Heidegger, "[...] est entièrement sans rapport au monde ambiant [umweltlich ganz unbezogen ist] $»^{58}$. «Les actions passées, même les tentatives mal réussies et les résultats ratés, aussi bien que les rencontres, les circonstances et les habitudes» peuvent certainement «jouer un rôle», ils ne constituent pas pour autant le noyau ou, pourrions-nous dire, le cœur de l'ipséités9. Voici le résultat le plus remarquable auquel nous parvenons: le se donner du «je», son évidence, ne coïncide pas nécessairement avec son être là. Le Dasein, ici opposé à l'Existenz - et la terminologie de ces premiers cours est encore loin d'une sédimentation définitive - se distingue du simple «da sein $»^{60}$. «Der Mensch kann da sein, Dasein haben ohne zu existieren $»^{61}$. «L'homme», affirme Heidegger, «peut être là, avoir l'être là sans exister» et, par reflet, l'« exister» exige un surplus eu égard au simple «être là». C'est seulement en détruisant la facticité que nous obtenons «l'existence», où celui qui existe, n'est pas, il n'a pas les caractères du monde, mais $a$ soi-même.

La confrontation avec Natorp s'établit, essentiellement, sur cet acquis, en marquant une étape et à la fois l'ouverture d'un parcours - il est à peine besoin de le dire - bien plus vaste: de l'initiale Lebensphilosophie jusqu'à la célèbre Seinsfrage, la réflexion ira en évoluant progressivement, non sans variations de taille ni changements d'orientation. Et ce sont les questions laissées inévitablement en suspens qui définiront l'orientation de la marche à venir. Il reste en fait à comprendre le type exact d'expérience, existentiellement entendue, en vertu de laquelle l'ipséité en se renouvelant devient soi-même ${ }^{62}$. Une fois attesté le «comment» qui en définit, si l'on peut dire, le modèle formel,

${ }^{58}$ Ibid.

${ }^{59}$ Cfr. GA 59, p. 81.

${ }^{60}$ A propos du statut ontologique du terme «Dasein» et sa progressive institution, cf. cfr. J. Kisiel, «The missing link in the Early Heidegger», in J.J. Kockelmans (ed.), Hermeneutic Phenomenology. Lectures and Essays, University Press of America, Washington D.C. 1988, p. 21: «[...] although it [i.e. Dasein] begins to be equated with "life" already in WS 1921-22 (e.g., on p. 85 of GA 61) and in October 1922, i.e., with the ontologizing of terms that sets in with Heidegger's turn to Aristotle, "Da-sein" does not receive official status until SS 1923, when it is introduced to underscore the temporal particularity (Jeweiligkeit) of "my" life».

${ }^{61}$ GA 59, p. 82; tr. it., p. 71.

${ }^{62}$ C'est là la question que l'on retrouvera, et non par hasard, dans Sein und Zeit. Cf. GA 2, § 25, p. 117. 
il s'agit d'en saisir concrètement le «temps» ${ }^{63}$. Comment j'existe, quand j'existe? La réponse, dans les cours de 1919-20, s'esquisse plutôt qu'elle ne se précise. Quelques indications extrêmement significatives sont néanmoins à noter, dans lesquelles Heidegger se confronte au corpus de la tradition biblique, à la recherche d'une solution phénoménologique au problème de l'ipséité. «Paul», lisons-nous dans le cours du semestre d'hiver de 1920-21, «thématise l'effectuation» ${ }^{64}$. La référence va spécialement à la première lettre aux Thessaloniciens et à l'annonciation de la «parousie», la seconde venue du Messie. La question est, alors, quand viendra-t-il? La métaphore de Paul est célèbre: «comme un voleur dans la nuit ainsi viendra le Seigneur»" ${ }^{65} \mathrm{La}$ réponse au «quand» s'articule dans la temporalité du «comment»: « comme vous n'êtes pas dans les ténèbres, ce jour ne vous surprendra pas comme un voleur $»^{66}$. Le chrétien vit dans la parousie, l'annonciation de la fin des temps, et c'est dans son attitude, le mode d'après lequel il vit, que s'effectue le sens de l'attente - le «quand» est un premier lieu une expérience, c'est-à-dire un témoignage de vie. Dans la première lettre aux Corinthiens, nous trouvons une autre indication: «frères, le temps est limité, dès lors $[\ldots]\rangle^{67}$. L'annonce de la venue fixe une «heure», l'instant, tò võv, où le Vollzug s'accomplit: dès lors, écrit Paul, soyez comme si non - $\omega \varsigma$ $\mu$ ń: «que ceux qui ont une femme soient comme s'ils n'avait pas de femme; ceux qui pleurent, comme $\mathrm{s}^{\prime}$ ils ne pleuraient pas, ceux qui ont de la joie, comme s'ils n'en avaient pas ${ }^{68}$.

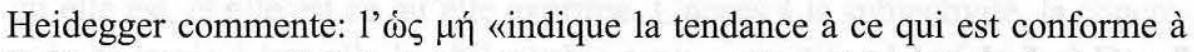
l'effectuation», et il doit s'entendre en tant que dispositif phénoménologique œuvrant en vue de l'ipséité ${ }^{69}$. Mais comment exactement? En faisant en sorte que la significativité de la vie réelle, tout en demeurant la même, change de sens. Les choses là dehors restent identiques - les arbres sont arbres, les maisons maisons... Le chrétien ne sort pas du monde; il vit, souffre, aime dans le monde sans pourtant - et voilà ce qui importe-se soucier du monde. Le sens de cette indication est complété par un passage de la lettre aux Galates: « de

${ }^{63}$ Voir à ce propos le développement ultérieure et la conséquente ontologisation du terme «Vollzug», assimilé à «Zeitung», après le «tournant aristotélicien»: M. Heidegger, Phänomenologische Interpretationen zu Aristoteles. Einfürhung in die phänomenologische Forschung, GA 61, p. 53. Pour un approfondissement, nous renvoyons à C. Sommer, «L'inquiétude de la vie facticielle: le tournant aristotélicien de Heidegger (1921-1922)», in Les études philosophiques, I, 2006, p. 1-28.

${ }^{64}$ GA 60, p. 121.

${ }^{65} 1 \mathrm{Ts}, 5,2$.

${ }^{66} 1 \mathrm{Ts}, 5,4$.

${ }^{67} 1 \mathrm{Cor}, 7,29$.

${ }^{68} 1$ Cor, 7, 29-30.

${ }^{69}$ GA 60, p. 121; tr. it., p. 151. 
même nous aussi [...] nous étions en situation d'esclaves, soumis aux forces qui régissent le monde» - «esclaves», «mais lorsqu'est venue la plénitude des temps $[\ldots]\rangle^{70}$. La plénitude des temps, c'est-à-dire la plénitude du soi en tant que temps de l'attente, qui est également le temps de la fin, le temps qui ne se superpose pas aux «jours, aux mois et aux années» ${ }^{71}$, qui ne s'aplatit pas sur la quotidienneté familière le long de laquelle les habitudes se sédimentent et la vie, silencieusement, s'établit, se rassure, en se rendant invisible à ellemême. "“Quelle paix” ! "Quelle tranquillité" !, c'est alors que, tout à coup, la catastrophe s'abattra sur eux» ${ }^{72}$ - Heidegger, pour sa part, parlera de Ruinanz, en entendant par là la chute de l'existence, l'usure de la significativité originaire. Dans le dispositif du «comme si non» s'exprime le principe de la méthode destructrice, en vertu duquel s'accomplit l'élan phénoménologique vers l'ipséité - l'ipséité qui ne peut jamais s'assimiler pleinement à ce qui est dans le monde; comme si elle $n$ 'était pas dans le monde et, donc, en un sens, comme si elle n'était «rien». En suspendant tout renvoi, le soi ne renvoie plus à rien d'autre qu'à lui-même. Expérience de soi comme effectuation du soi, dont la plénitude invoque un temps fini, présent et toujours à venir, et où l'être le plus propre, la fin, coïncide avec un être pour la fin - c'est-à-dire, comme Heidegger le retraduira peu après, en conjuguant Luther avec Aristote, un «être-pour-la-mort».

\section{Conclusions}

«Facticité et existence ne signifient pas la même chose» ${ }^{73}$. Les thèses de 1919-20 aboutiront, élaborées ultérieurement, dans cet écrit programmatique qui va sous le titre d' «Interprétations phénoménologiques d'Aristote» et dont Natorp fut, en 1922, le destinataire enthousiaste. Tellement enthousiaste qu'il n'hésitera pas à retrouver dans la perspective du jeune Heidegger l'essentiel de ses propres thèses ${ }^{74}$. Dans cette version inédite de la phénoménologie, et

${ }^{70} \mathrm{Gal}, 4,3-4$.

${ }^{71} \mathrm{Gal}, 4,10$.

${ }^{72} 1 \mathrm{Ts}, 5,3$.

${ }^{73}$ M. Heidegger, Phänomenologische Interpretationen zu Aristoteles (Anzeige der hermeneutischen Situation), in Dilthey-Jahrbuch, 6, (1989), pp. 235-274; Phänomenologische Interpretationen zu Aristoteles. Ausarbeitung für die Marburger und die Göttinger Fakultät (1922), Reclam, Stuttgart, 2003 (rist. in GA 62); trad. fr. J-F. Courtine, Interprétations phénoménologiques d'Aristote, T.E.R., Mauvezin 1992.

${ }^{74}$ Cf. la lettre de Natorp à Husserl du 9. XI. 1922, in Edmund Husserl: Briefwechsel, vol. V, hrsg. K. Schuhmann - E. Schuhmann, Kluwer 1994, p. 162-163, cit. par J. Kisiel, « The missing link in the Early Heidegger », cit., p. 14. Natorp, toutefois, comme Kisiel remarque, 
avant même que le «tournant aristotélicien» impose à la recherche un horizon définitivement ontologique, le point de départ et le point d'arrivée sembleraient alors coïncider - étrangement, mais significativement - avec la disposition de l'Allgemeine Psychologie. Qu'il soit transcendantal ou existentiel, le «je» ne se charge pas des attributs de la facticité. «Reconstruisant», dans un cas, ou «détruisant», dans l'autre, nous remontons de toute façon à rebours vers le sens d'une origine qui ne se donne jamais, directement, pour ce qu'elle est. Le commencement est nécessairement négatif, comme négatif est le rapport entre le je et le monde. Auprès des choses nous nous trouvons, auprès des choses nous nous perdons. Et c'est seulement par un contre-mouvement qui renverse le cours normal de la vie, que nous pouvons devenir une question à nous-mêmes - Quaestio mihi... De même pour la superposition qui vient s'établir entre la méthode et le thème: que ce soit par effectuation ou par constitution, la réponse au qui s'articule dans un comment et c'est dans le comment que doit se repérer le sens, éminemment temporel, de la subjectivité. «Toutes les questions de la philosophie» - Heidegger commente à son tour «au demeurant ne sont que des questions concernant le "comment", c'est-àdire, dans un sens étroit, des questions concernant la méthode» ${ }^{75}$.

La subjectivité, son sens, pourrions-nous conclure, coïncide essentiellement avec son accès, avec la méthode elle-même - avec le sens d'une effectuation, le Vollzug, comme si elle n'était qu'une performance qui exprime ce qu'elle est, et elle est ce qu'elle exprime. L'accès à la subjectivité, la concrétude de la vie à laquelle la philosophie aspire, constitue à la fois le thème et la méthode de la philosophie elle-même. Subjectivité et philosophie, l'une et l'autre coïncident essentiellement et cette coïncidence porte le nom de «phénoménologie». «Pour nous» - reconnaît encore Heidegger - «à l'arrière-plan se trouve la thèse que la philosophie n'est pas une science théorétique» ${ }^{76}$. Et c'est à la lumière de cette originalité que se mesure la distance vis-à-vis de Natorp, nonobstant les signes d'une reconnaissance encore plus profonde: «la position de Natorp est tout autant éloignée de l'origine, d'après notre sens distante et pervertie, que sa recherche de l'origine, selon son propre sens, est

n'explicite pas d'avantage les raisons de cette surprenante affinité, et le même Kisiel commente ainsi: «What was it about Heidegger's burgeoning ideas that reminded Natorp of his own work? This could be the subject of a dissertation, but one can at least mention that, in his lecture courses since 1917, Natorp out of his neo-Kantian framework had been discussing the dynamic fieri of facticity and made some use of the etymological sens of aletheia as unconcealment». Cf. également H. Ott, Martin Heidegger. Unterwegs zu seiner Biographie, Campus, Frankfurt-New York 1988, p. 122.

${ }^{75}$ GA 60, p. 88.

${ }^{76}$ GA 60, p. 62. 
intense et radicale ${ }^{77}$. Le pas initial auquel nous assistons dans ces premiers cours de Fribourg est en réalité un pas en avant extrêmement ambitieux, qui entend assurer à l' " ipséité» une consistance phénoménologique: «le je ne peut pas être pensé comme un je, il ne peut aucunement être un objet de pensée», pourtant - et c'est ici que demeure le motif de l'opposition - «la philosophie ne renonce pas à la pensée» ${ }^{78}$. En se démontrant fidèle au principe de la $\mathrm{Ge}$ gebenheit, contre Natorp, le jeune Heidegger conclut alors: le soi s'effectue, c'est-à-dire se donne par effectuation. Comment? En vertu d'une tension entre facticité et existence, une tension qui confirme ainsi, et à nouveaux frais, la question d'où nous sommes partis - dans quel sens celui qui existe existe-t-il?

\section{RÉSUMÉ}

Au sein de la Seinsfrage s'annonce la question du qui, la Werfrage. Qui est ce «qui»? A savoir qui suis-je? En questionnant l'être, c'est inévitablement nous-mêmes que nous questionnons. Serait-il alors possible de penser le sujet en dehors ou à la rigueur avant l'être? Cette question, en apparence paradoxale, a pourtant un fondement historiographique, si l'on considère que l'«anthropologie phénoménologique», telle que le jeune Heidegger la conçoit, précède de fait la formulation de la Seinsfrage. Dès les premiers cours de Fribourg, est ainsi dégagée une voie d'accès vers le sujet, entendu comme «ipséité mondaine». Tout en critiquant Natorp, qui sans réserve niait une telle possibilité, Heidegger finit pourtant par lui concéder l'essentiel. C'est du moins notre hypothèse de recherche: le je n'existe qu'au prix d'une tension entre facticité et existence, une tension qui aboutit, aux yeux de Natorp, à confirmer que le je, à proprement parler, n'est pas - ce qui nous laisserait dès lors entrevoir la légitimité d'une Werfrage sans Seinsfrage.

Mots clés: Heidegger, Natorp, sujet, je, facticité, existence, destruction, reconstruction, Vollzug.

\section{ABSTRACT}

Within the Seinsfrage, the question of being, the «who» question, or Werfrage, arises at once. Who is this «who»? In other words, who am I? Questioning the being, inevitably means questioning ourselves. Would it thus be possible to conceive of a subject without being? This question, paradoxical as it may appear, nevertheless has a historiographical legitimacy, if we consider that «phenomenological anthropology», as the young Heidegger conceived it, precedes in effect the formulation of the Seinsfrage.

77 GA 59, p. 96.

${ }^{78}$ GA 59, p. 144. 
From his very first lectures in Fribourg, an access into subjectivity, apprehended as a "wordly ipseity ", is thereby opened. While criticizing Natorp who unreservedly denied such a possibility, Heidegger yet ends up conceding the point almost entirely. This, at least, is our hypothesis: the «I» exists only at the cost of a tension between facticity and existence, a tension which, in the eyes of Natorp, leads to a confirmation that the «I», strictly speaking, cannot be - and this allows us therefore a glimpse into the legitimate possibility of a Werfrage without Seinsfrage.

Keywords: Heidegger, Natorp, subject, I, facticity, existence, destruction, reconstruction, Vollzug. 